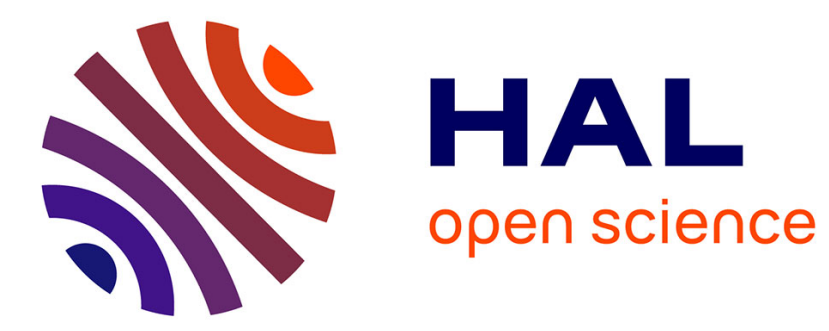

\title{
On the Role of Tsallis Entropy in Image Processing Amelia Carolina Sparavigna
}

\section{To cite this version:}

Amelia Carolina Sparavigna. On the Role of Tsallis Entropy in Image Processing. International Scientific Research Journal , 2015, 1 (6), pp.16-24. 10.18483/IRJSci.79 . hal-01352704

\section{HAL Id: hal-01352704 https://hal.science/hal-01352704}

Submitted on 9 Aug 2016

HAL is a multi-disciplinary open access archive for the deposit and dissemination of scientific research documents, whether they are published or not. The documents may come from teaching and research institutions in France or abroad, or from public or private research centers.
L'archive ouverte pluridisciplinaire HAL, est destinée au dépôt et à la diffusion de documents scientifiques de niveau recherche, publiés ou non, émanant des établissements d'enseignement et de recherche français ou étrangers, des laboratoires publics ou privés. 


\title{
On the Role of Tsallis Entropy in Image Processing
}

\author{
Amelia Carolina Sparavigna \\ Department of Applied Science and Technology, Politecnico di Torino, Torino, Italy.
}

\begin{abstract}
In image processing, the maximum entropy principle is generally recognized as having a relevant role in the initial part of image elaboration. The first step of processing in fact, sees the entropy used to determine the segmentation of the image, that is, used to determine objects and background in it. Different entropy formulations are available to this purpose, but the most prominent in recent publications is that of the Tsallis non-extensive entropy. Here, we survey some main methods that are using this entropy and the related literature, in particular that reporting the researches concerning medical image processing.
\end{abstract}

Keywords: Tsallis Entropy; Image Processing; Image Segmentation; Image Thresholding; Medical Image Processing

\section{Introduction}

Aiming to formulate a generalization of classical statistics which could be suitable for nonextensive thermodynamics, Constantino Tsallis postulated in 1988 a new expression of the entropic function [1]. Inspired by some multifractals concepts, Tsallis proposed an entropy that depends on a dimensionless parameter such as the scaling functions of universal multifractals are depending on a multifractality index [1-3]. This parameter is usually indicated by $q$; in the limit $q \rightarrow 1$, Tsallis entropy is recovering the expression of Boltzmann-Gibbs-Shannon entropy.

Today, we have that a strongly increasingly number of natural and artificial systems, including the social systems too, are studied by means of Tsallis entropy [4]. To evaluate how its role increased during the years, we can easily use a web search engine indexing the scholarly literature, Google Scholar for instance, applying it to proper custom ranges. If we make this search, we can also appreciate that, in the last five years, of all the total researches referring to this entropy, most of them are on its use in image processing.

Tsallis entropy enters the image processing through the image segmentations made by means of the maximum entropy principle. Segmentation is the processing task that separates the pixels in those pertaining to objects and in those pertaining to the background; it is then the important first step that helps identifying the image components and that allows extracting their features [5]. In segmentation, the methods that are using the maximum entropy principle are generally privileged, because they are considered consistent methods allowing the incorporation of extra knowledge about the objects represented in the image [6].

Before the end of the last century, several different methods existed for image thresholding based on an entropy maximization. Some of these methods used the gray level histograms, while others used two-dimensional histograms or co-occurrence matrices, involving the Shannon entropy. However, some researchers, like authors in [7], started investigating the use of the "non-Shannonian" entropies. Since most of these entropies have parameters that can influence the performance of the algorithms, their use was considered quite relevant for further development.

In 2004, the first paper based on Tsallis entropy for image thresholding was published [8]. From this first publication, the number of researches on image processing with Tsallis entropy started growing. Today, a large part of segmentation methods is based on this entropy, which proved being the best possible choice. Here, we survey the methods using it and the related literature, from which we 
can clearly appreciate as Tsallis entropy is becoming the corner stone of medical and fuzzy image processing.

\section{Tsallis entropy and its non-additivity}

Since images are composed of pixels having discrete gray levels, our discussion will be made through a discrete set of probabilities $\left\{p_{i}\right\}$, with random variable $i$. Condition on probabilities is: $\sum_{i} p_{i}=1$. For any real parameter $q$, Tsallis entropy is defined as:

$$
S_{q}=\frac{k}{q-1}\left(1-\sum_{i} p_{i}^{q}\right)
$$

Sometimes, parameter $q$ appearing in (1) is named the "entropic index"; $k$ is a constant that in the image processing is set to 1 . In the limit $q \rightarrow 1$, the Boltzmann-Gibbs-Shannon (BGS) entropy is recovered. This is the classical entropy used for systems having short-range microscopic interactions and microscopic memory. Systems obeying BGS statistics are extensive systems.

Let us consider a physical system decomposed into two independent systems $A$ and $B$, having joint probability: $p(A, B)=p(A) p(B)$, BGS entropy is additive:

$$
S_{B G S}(A, B)=S_{B G S}(A)+S_{B G S}(B)
$$

In the case Tsallis entropy is evaluated, we have:

$$
S_{q}(A, B)=S_{q}(A)+S_{q}(B)+(1-q) S_{q}(A) S_{q}(B)
$$

In the appendix of Ref.9, the reader can find a detailed discussion on this generalized additivity. Tsallis entropy possesses the remarkable property of being non-additive, with the modulus $|1-q|$ measuring the departure from additivity. In the limit of $q \rightarrow 1$, we have the expected additivity of entropy. In the case $q<1$, the entropy is sub-extensive: $S_{q}(A, B)<S_{q}(A)+S_{q}(B)$. When $q=1$, it is extensive and when $q>1$, it becomes super-extensive: $S_{q}(A, B)>S_{q}(A)+S_{q}(B)$.

In fact, Tsallis entropy is linked to the entropy proposed by Alfred Rényi, which is defined as [10]:

$$
\bar{S}_{q}\left(p_{i}\right)=\frac{1}{1-q} \ln \left(\sum_{i} p_{i}^{q}\right)
$$

This entropy is additive and the parameter $q$ is used to have it more or less sensitive to the shape of probability [11]. The link between Tsallis and Rényi entropy is given in [1]:

$$
\bar{S}_{q} \equiv \frac{1}{1-q} \ln \left[1+(1-q) S_{q}\right]
$$

Let us assume two independent systems $A$ and $B$ again. The Rényi entropy is additive, so that:

$$
\bar{S}_{q}(A, B)=\bar{S}_{q}(A)+\bar{S}_{q}(B)
$$

From (5) and (6), we easily obtain (3). Let us remember also that in 1967, Havrda and Charvát defined an entropy [12]: 


$$
S_{q}^{H C}=\frac{1}{1-2^{1-q}}\left(1-\sum_{i} p_{i}^{q}\right)
$$

This entropy differs from that proposed independently by Tsallis for the normalization factor. The Havrda and Charvát entropy is normalized to 1, whereas the Tsallis entropy is not normalized. As observed in [13], in image thresholding, both entropies yield the same result and, for this reason, the entropy is also named Tsallis-Havrda-Charvát entropy.

\section{Entropy and image segmentation}

A common process used to segment an image is the thresholding, usually classified as bi-level and multi-level thresholding. In the bi-level thresholding, the pixels are separated into two classes, whereas, in the multi-level thresholding, the separation is generalized to several classes [5].

In [14], a survey of thresholding is given, which is categorizing the methods into some groups based on the information the algorithms are manipulating. Among them, we find methods based on histograms of the gray-level sample or methods based on clustering, where the gray-level samples are clustered in two parts as background and objects. We have also the entropy-based methods: Kapur et al. [15] for instance, assumed two probability distributions, one for the object and the other for the background and maximized the total entropy of the partitioned image in order to obtain the threshold level. In [8], the authors used a method similar to the maximum entropy method [15], but applying Tsallis entropy. To the best of our knowledge, they were the first researches that used this entropy in image processing.

Let us consider an image having $k$ gray levels. A digital image of size $X \times Y$ is a matrix of the form $[f(x, y) \mid x=1,2, \ldots, X ; y=1,2, \ldots, Y]$, where $f(x, y)$ is the gray value of pixel located at point $(x, y)$. The set of all gray values $\{0,1,2, \ldots, k\}$ usually has $k=255$. We need a distribution of probabilities, $p_{1}, p_{2}, \ldots, p_{k}$ for the gray levels: for it, we can use the normalized histogram $h_{i}=n_{i} / n_{\text {tot }} ; n_{i}$ is the number of pixels with gray value $i$ and $n_{\text {tot }}$ is the number of all pixels in the image (in Figure 1, an example of histogram).
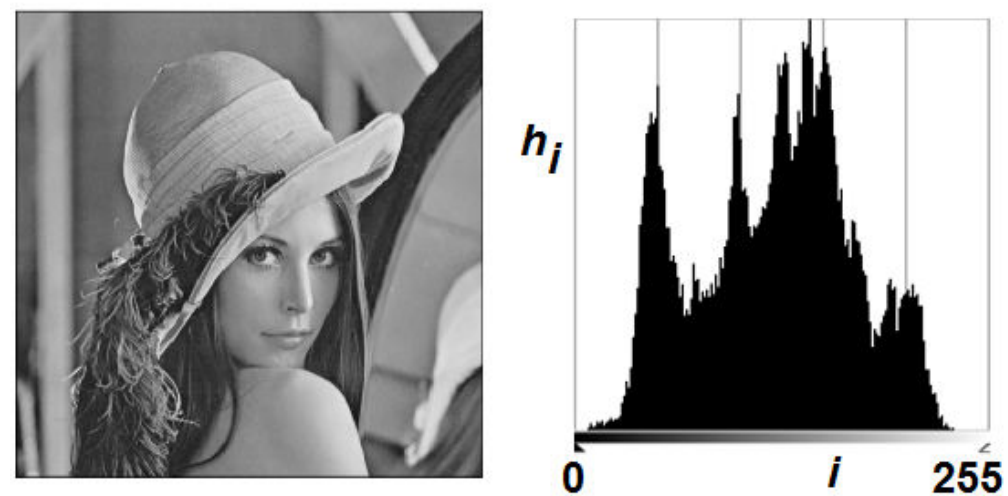

Figure 1 - The image shows lena.jpg (royalty free image) in gray tones. On the right, we can see its histogram, obtained using GIMP (GNU Image Manipulation Program).

Let us assume a bi-level threshold $t$ for the gray levels. In [8], two classes had been introduced, $A$ and $B$, and their probability distributions:

$$
A: \frac{p_{1}}{p_{A}}, \frac{p_{2}}{p_{A}}, \ldots, \frac{p_{t}}{p_{A}} ; B: \frac{p_{t+1}}{p_{B}}, \frac{p_{t+2}}{p_{B}}, \ldots, \frac{p_{k}}{p_{B}} ; p_{A}=\sum_{i=1}^{t} p_{i} ; p_{B}=\sum_{i=t+1}^{k} p_{i}
$$

The Tsallis entropies, one for each distribution, are given by: 


$$
S_{q}^{A}(t)=\frac{1}{q-1}\left\{1-\sum_{i=1}^{t}\left(\frac{p_{i}}{p_{A}}\right)^{q}\right\} ; S_{q}^{B}(t)=\frac{1}{q-1}\left\{1-\sum_{i=t+1}^{k}\left(\frac{p_{i}}{p_{B}}\right)^{q}\right\}
$$

The total entropy is:

$$
S_{q}(t)=S_{q}^{A}(t)+S_{q}^{B}(t)+(1-q) S_{q}^{A}(t) S_{q}^{B}(t)
$$

When this entropy, which is a function of threshold $t$, is maximized, the corresponding gray level $t$ is considered the optimum threshold value [8]. Of course, this threshold is depending on $q$ parameter. An example of thresholding is given in Figure 2 on lena.jpg. In the same image, we can see also the Tsallis entropy as a function of the chosen threshold. Since the final result depends on $q$, this parameter can be used as an adjustable value and can play an important role as a tuning parameter [8].

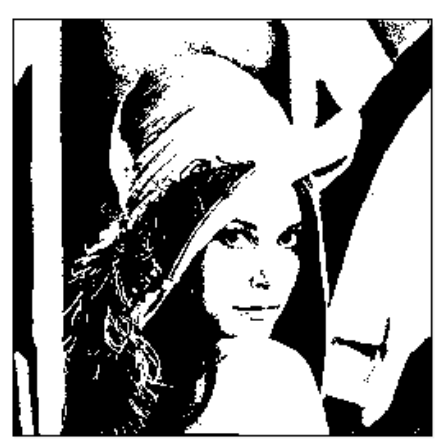

$\mathrm{t}=120, q=0.50$

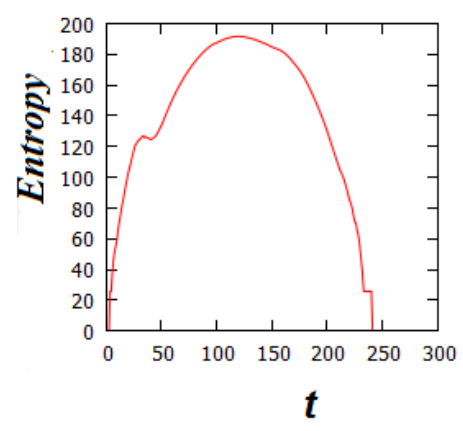

Figure 2 - Effect of a thresholding, obtained by maximizing Tsallis entropy. Pixels having a gray tone larger than the threshold become white; pixels having a lower value become black. Tsallis entropy as a function of threshold is also given. The maximum value gives the threshold, in this case, $t=120$. Changing parameter $q$, the threshold is different, such as the values of entropy.

Rényi entropy based image thresholding [16,17] and Tsallis entropy based image thresholding [8] are two important approaches to the global threshold selection for image segmentation. In fact, they can provide equivalent results, that is, the two approaches give the same threshold when the same parameter is chosen.

In computer vision and image processing, the reduction of a gray level image to a binary image is often used, but we can find also a multi-level thresholding. Let us consider again an image having $k$ gray levels, but several thresholds $t_{1}, t_{2}, \ldots, t_{m}$. In this case, $(m+1)$ classes must be introduced and the corresponding probability distributions. Generalizing Equations 8-10 (for all details, see please Ref.18), the Tsallis entropies for each distribution and the total entropy are:

$$
\begin{aligned}
& S_{q}^{(1)}=\frac{1}{q-1}\left\{1-\sum_{i=1}^{t_{1}}\left(\frac{p_{i}}{P_{1}}\right)^{q}\right\} ; \cdots ; S_{q}^{(m+1)}=\frac{1}{q-1}\left\{1-\sum_{i=t_{m}+1}^{k}\left(\frac{p_{i}}{P_{m+1}}\right)^{q}\right\} \\
& S_{q}=\sum_{i=1}^{m+1} S_{q}^{(i)}+(1-q) \frac{1}{2 !} \sum_{i=1}^{m+1} \sum_{j=1 ; j \neq i}^{m+1} S_{q}^{(i)} S_{q}^{(j)}+(1-q)^{2} \frac{1}{3 !} \sum_{\substack{i=1 \\
m+1}}^{m+1} \sum_{\substack{j=1 \\
j \neq i}}^{m+1} S_{\substack{k=1 \\
k \neq j, k \neq i}}^{(i)} S_{q}^{(j)} S_{q}^{(k)}+\cdots
\end{aligned}
$$


The last term in (12) is $(1-q)^{m} S_{q}^{(1)} S_{q}^{(2)} \cdots S_{q}^{(m)} S_{q}^{(m+1)}$.

In [19], the authors proposed a multi-level thresholding method based on Tsallis entropy for image segmentation. They used an interesting artificial bee colony approach to reduce the time of processing. In [20], a multi-level thresholding was proposed too, but this is combining Tsallis entropy and Particle Swarm Optimization (PSO). PSO is a computational method of optimization, which use iterative tests to improve a candidate solution. The same was made in [21].

In the Figure 3, an example of multi-level thresholding (three levels) using the Tsallis entropy is given. For the image, we optimized two thresholds. In the same Figure we can see the behavior of entropy: in dark gray we have the entropy as a function of threshold $t_{1}$ for different values of the other threshold; in light gray, we have the same for threshold $t_{2}$. In the Figure 4, we have the comparison of lena.jpg after bi-, three- and four-level thresholding.
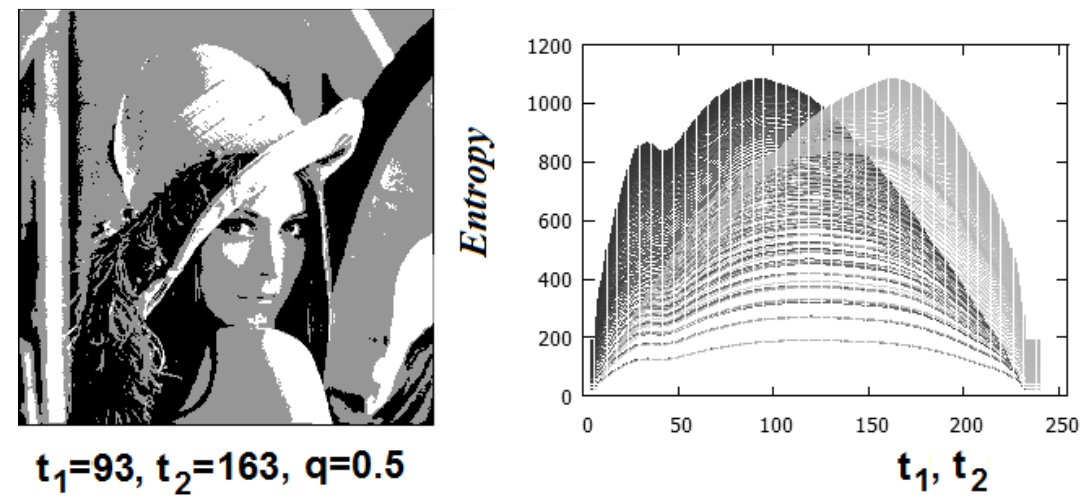

Figure 3 - An example of multi-level thresholding using Tsallis entropy (three levels) on lena.jpg. We can see also the behavior of the entropy: in dark gray we have the entropy as a function of threshold $t_{1}$, for different values of the other threshold; in light gray, we show the same for threshold $t_{2}$.

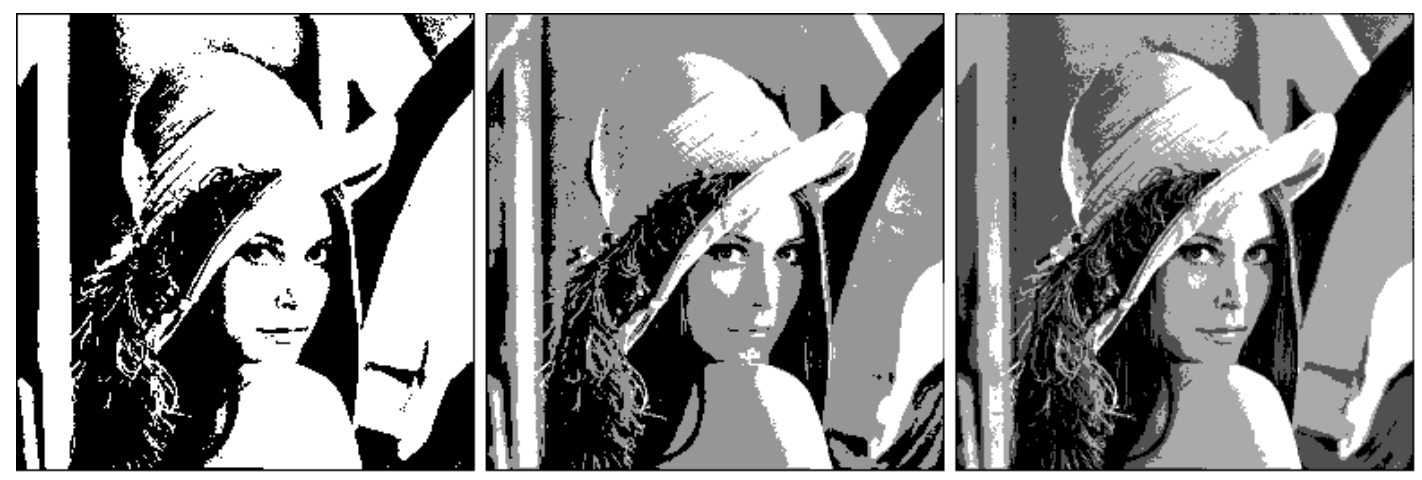

Figure 4 - Bi-level, three-level and four-level thresholding on lena.jpg.

\section{Two-dimensional Tsallis entropy}

In the introduction, it had been told that segmentation can be obtained also by means of a twodimensional histograms. First of all, let us see what a two-dimension histogram is. We have already defined $f(x, y)$ as the gray value of the pixel located at point $(x, y)$. Besides this function we can use the average gray value, $g(x, y)$, of the pixel's neighborhood too. The function is obtained calculating the integer part of the arithmetic mean of nine gray tones, which are the tone of the given pixel and the tones of its eight nearest neighboring pixels. The gray value of the pixel, $f(x, y)$, and the average of its neighborhood, $g(x, y)$, are used to build a two-dimensional histogram. The probability is given by the 
normalized histogram $h(i, j)=n_{i j} / n_{t o t}$, where $n_{i j}$ is the number of pixels with gray value $i$ and average gray value $j$, and $n_{\text {tot }}$ is the number of pixels in the image.

Again, we need distinguishing object and background. The threshold is obtained through a vector $(t, s)$ where $t$, for $f(x, y)$, represents the threshold of the gray level of the pixel and $s$, for $g(x, y)$, represents the threshold of the average gray level of the pixels neighborhood. In this manner, we find a histogram creating a surface that will have two peaks and one valley [13]. The object and background correspond to the peaks and can be separated by selecting the proper vector $(t, s)$ that maximizes a suitable criterion function $U(t, s)$. Using vector $(t, s)$, the domain of the histogram is divided into four quadrants: $[0, t] \times[0, s],[0, t] \times[s+1, k],[t+1, k] \times[0, s]$ and $[t+1, k] \times[s+1, k]$. Two of the quadrants contain information about edges and noise alone, and therefore they are ignored in the calculation [13]. The quadrants which contain the object and the background are considered to have independent distributions. Their probability values must be normalized in order to have a total probability equal to 1. The normalization is accomplished by using a posteriori class probabilities:

$$
P_{2}(t, s)=\sum_{i=0}^{t} \sum_{j=0}^{s} h(i, j) ; P_{4}(t, s)=\sum_{i=t+1}^{k} \sum_{j=s+1}^{k} h(i, j)
$$

If the contribution of the quadrants which contains edges and noise is assumed negligible, we can approximate $H_{4}(t, s)=1-H_{2}(t, s)$. Distributions and entropies are:

$$
\left\{\begin{array}{l}
A: \frac{h(0,0)}{P_{2}(t, s)}, \ldots, \frac{h(0, s)}{P_{2}(t, s)}, \frac{h(1,0)}{P_{2}(t, s)}, \ldots, \frac{h(t, s)}{P_{2}(t, s)} \\
B: \frac{h(t+1, s+1)}{P_{4}(t, s)}, \ldots, \frac{h(t+1, k)}{P_{4}(t, s)}, \frac{h(t+2, s+1)}{P_{4}(t, s)}, \ldots, \frac{h(k, k)}{P_{4}(t, s)}
\end{array}\right.
$$

Entropies and total entropy are:

$$
\begin{aligned}
& S_{q}^{A}(t, s)=\frac{1}{q-1}\left\{1-\sum_{i=1}^{t} \sum_{j=1}^{s}\left(\frac{h(i, j)}{P_{2}(t, s)}\right)^{q}\right\} \\
& S_{q}^{B}(t, s)=\frac{1}{q-1}\left\{1-\sum_{i=t+1}^{k} \sum_{j=s+1}^{k}\left(\frac{h(i, j)}{P_{4}(t, s)}\right)^{q}\right\} \\
& S_{q}(t, s)=S_{q}^{A}(t, s)+S_{q}^{B}(t, s)+(1-q) S_{q}^{A}(t, s) S_{q}^{B}(t, s)
\end{aligned}
$$

This entropy is a function of thresholds $t$ and $s$; it is also known as the two-dimensional, or 2D, Tsallis entropy [22]. In the reference, the effectiveness of the approach was demonstrated by using examples from the real-world and synthetic images, concluding from the experimental results, that the method gets better results when using the Tsallis entropy rather than the Shannon entropy.

\section{Automated segmentation and edge detection}

The segmentation methods we have previously discussed reveal, as shown by the experimental results proposed in literature, a larger efficiency when used with Tsallis entropy. Therefore, not surprisingly, this entropy has become fundamental for automated segmentation and tracking. This is an important task, for instance, for monitoring the motion of cells in culture, in particular of hematopoietic stem cells (HSCs), which proliferate and differentiate to different blood cell types during their life [23]. For such a monitoring, reliable automatic methods are requested, because, if 
undertaken manually, researchers are compelled to visually perform the analysis, observing cell movements and cell shape changes for hours and hours [23].

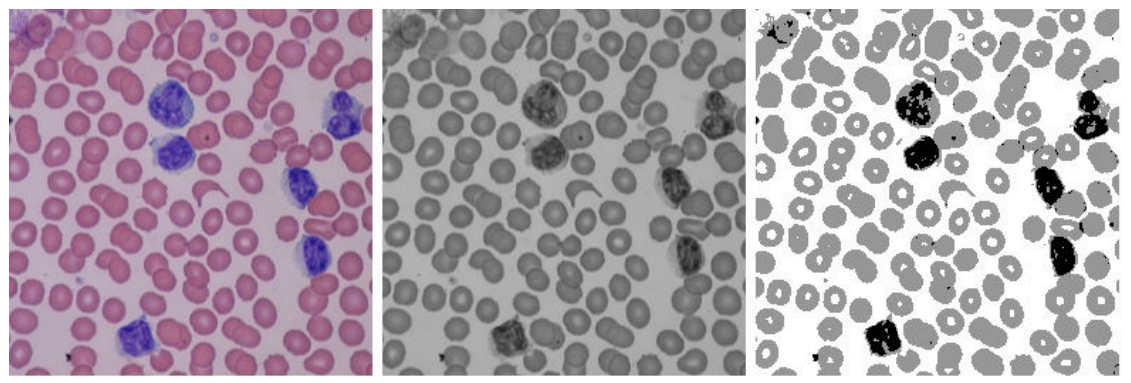

Figure 5 - A three-level thresholding on a light microscope image (Courtesy Wikipedia) of blood cells. The color image is converted in gray tones. The final image on the right is given after using Tsallis entropy. Note that levels correspond to different types of cells and background. The processing is made with $q=0.5$.

In the Figure 5, we can see an example of a three-level thresholding based on Tsallis entropy applied to an image of blood cells. Here, we have the occasion of showing the role of parameter $q$. Its right choice becomes fundamental for detecting the blood cells, for instance. We can appreciate this fact observing the following experiment on the same image of Figure 5. If we change parameter $q$ in the range [0,1], we see the visibility of red cells disappearing when $q$ is greater than 0.66 (Figure 6).

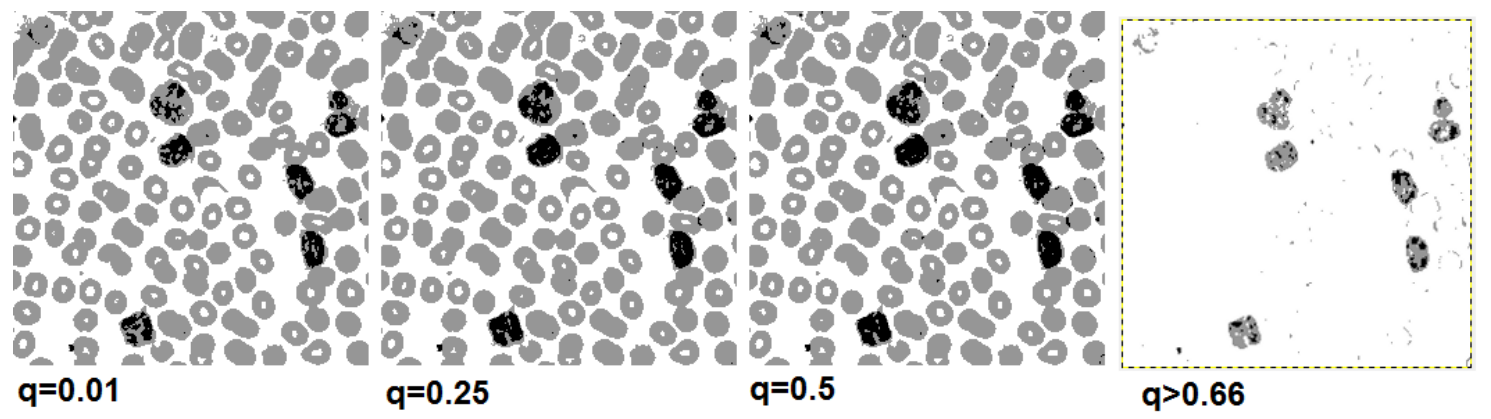

Figure 6 - The three-level thresholding is depending on parameter $q$. In this example we can see that when $q>0.66$, the visibility of red cells disappears.

The simple example here proposed puts in evidence that we need, in the segmentation of an image, the proper choice of $q$. In [24], the authors proposed an automated method for determining $q$ parameter, employing a fuzzy image segmentation technique. The combination of Tsallis entropy and fuzzy approach improved the result of segmentation, reducing the error in the classification of objects.

Image segmentation can also be based on edge detection, a pre-processing step particularly required in medical image segmentation. Many edge-detection methods have been proposed in the past years, such as Canny, Sobel and Prewitt algorithms. As stressed in [25], these methods belong to the category of high pass filtering; for such a reason, they do not fit for the edge detection of some images coming from real applications, such as the medical images which are containing shadows and noise. After studying all traditional methods of edge detection, authors in [25] found an algorithm for noisy medical images based on both Tsallis and Shannon entropy combined together.

\section{Entropy in medical image segmentation}

The methods that we have shown in the previous section, bi- and multi-level thresholding and twodimensional histogram, are the most used in applying Tsallis entropy. These methods have assumed, in 
the last decade, a relevant role in the most dynamic branch of image processing, that of the medical image processing [26].

The medical image processing is based, among several other methods, on clustering the presence of unwanted lesions or regions in a noisy background and in highlights the edges of poorly illuminated images. This is fundamental to define the edges of different regions. As a starting point of analysis, a segmentation is often used; in [27], automatic segmentations, based on pulse-coupled neural network (PCNN) for medical images have been proposed (actually, a feature extraction method via PCNN and Tsallis entropy was first proposed in Ref.28 for face recognition). In Ref.27, the processing was made without selecting the PCNN parameters because the best result was determined by means of the Tsallis entropy. Let us remark that pulse-coupled networks are models which are proposed for their highperformance biomimetic image processing [27,28].

The most recent papers using the Tsallis entropy in medical image processing are ranging from thresholding to the problem of image registration, as we can see for instance from the references [2933]. Image registration is a process of transforming different sets of data into the same coordinate system. Actually, it is a hot topic because this process is very important for comparing different medical analyses, but, at the same time, it is a very difficult task that needs to be simplified and optimized. In [32], an algorithm for image registration, combining the particle swam optimization (PSO) technique and the sequential quadratic programming (SQP) method had been proposed.

Among the works using Tsallis entropy in medical imaging, let us also highlight those that are studying one of the most challenging problem this research field is facing: the detection of microcalcification clusters for early breast cancer diagnosis. In [33], Tsallis entropy is used, associated to a fuzzy technique for the optimal determination of parameter $q$. The authors tested this approach on various images; their results demonstrate that the method based on Tsallis entropy outperforms the two-dimensional non-fuzzy approach and conventional Shannon entropy partition approach [33]. In [34], the Tsallis entropy is involved with wavelets transform too, for detecting microcalcification.

\section{Facial expression recognition}

For what concerns the general role of Tsallis entropy in pattern recognition, Ref.35 compared the effectiveness of the Tsallis entropy over the classic BGS entropy, for a general pattern recognition, and proposes a multi- $q$ approach to improve the pattern analysis. Experiments in [35] show that, for pattern classification, the Tsallis entropy using the proposed multi- $q$ approach has great advantages over the BGS entropy, boosting image recognition rates by a factor of 3 . This happens because the Tsallis entropy for different $q$ (the multi- $q$ approach) encodes much more information from a given probability distribution than the BGS entropy.

In the huge field of pattern recognition we find also several researches on the facial expression recognition. A facial expression is a manifestation on our faces of affective states and cognitive activities, but also of our intentions and personalities. Of course, it plays a fundamental role in interpersonal non-verbal communication. Facial expressions, such as other gestures, can be analyzed by computers with software created for their automatic recognition. This is a software addressing the problem of detecting and categorizing static and dynamic features of facial deformations or of facial pigmentation [36]. In the abovementioned Ref.28, we can find the first attempt of exploring the potential of Tsallis entropy in handling the face recognition problem. Experiments made by the authors demonstrated that the use of this entropy and of PCNN can improve the classification rate. Since PCNN does not need pre-training, the calculation time are also reduced.

As explained in [37], facial expression recognition can be based on some sets of features extracted from the face images, such as the texture features and global appearance features [37]. It is in finding the first set of the two, that of texture features, that the Tsallis entropy can be used [37], in a method using local binary patterns (LPBs). A survey of LBPs is given in [38].

\section{Fuzzy Tsallis entropy}

Fuzzy set theory is an extension of conventional set theory, which is dealing with the concept of partial truth [39]. It was proposed by Lotfi Zadeh in 1965 [40], aiming to model ambiguity and uncertainty in complex systems. In recent years, the use of fuzzy logic has been extended to image 
processing $[41,42]$, producing the fuzzy image processing. As in the usual processing, its application requires object recognition and scene analysis, where the fuzzy approach can the one able to solve the difficulties due to inherent ambiguity and vagueness of image data [39].

The image data are transformed from the gray-level plane to the membership plane [39]. In this manner, a classical thresholding technique can, using the image fuzziness, produce a new thresholding technique. In [43], for instance, thresholding is obtained combining Tsallis entropy and fuzzy cpartition. The image to be segmented is firstly transformed into fuzzy domains using membership function. Then, the fuzzy Tsallis entropies are defined and the threshold is selected by finding a proper parameter combination of the membership function, which is maximizing the total fuzzy Tsallis entropy.

\section{Conclusions}

In the first part of the paper, we made a survey of three fundamental methods for using Tsallis entropy in image segmentation, proposing some examples too. For illustrating the methods, several references had been used; here, let us just mention [8], the first that used Tsallis entropy for the image bi-level thresholding. The following studies demonstrated that, in several cases, this entropy is the best we can use for determining the optimal threshold values by means of which an image can be segmented. In fact, as we have seen from a survey of literature, this entropy is so successful that it is becoming the keystone of a large part of methods used in medical image processing and fuzzy image processing.

\section{References}

1. Tsallis, C. (1988). Possible generalization of Boltzmann-Gibbs statistics. Journal of Statistical Physics, 52, 479-487. DOI: 10.1007/BF01016429

2. Hentschel, H.G.E.; Procaccia, I. (1983). The infinite number of generalized dimensions of fractals and strange attractors. Physica D, 8, 435-444. DOI: 10.1016/0167-2789(83)90235-X

3. Halsley, T.C.; Jensen, M.H.; Kadanoff, L.P.; Procaccia, I.; Shraiman, B.I. (1986). Fractal measures and their singularities: the characterization of strange sets. Phys. Rev. A, 33, 1141. DOI: 10.1103/PhysRevA.33.1141

4. Tsallis, C. (2009). Nonadditive entropy and nonextensive statistical mechanics - an overview after 20 years. Braz. J. Phys., 39, 337-356. DOI: 10.1590/S0103-97332009000400002

5. Djerou, L.; Khelil, N.; Dehimi, N.H.; Batouche, M. (2012). Automatic multi-level thresholding segmentation based on multi-objective optimization. Journal of Applied Computer Science \& Mathematics, 13, 24-31.

6. Gull, S.F.; Skilling, J. (1984). Maximum entropy method in image processing. Communications, Radar and Signal Processing, IEE Proceedings F, 131, 646-659. DOI: 10.1049/ip-f-1.1984.0099

7. Mandal, D.P.; Pal, N.R. (2003). On the utility of different entropy measures in image thresholding. In Entropy Measures, Maximum Entropy Principle and Emerging Applications. Karmeshu, Ed. Springer Science \& Business Media, Volume 119, pp. 177-198. ISBN 978-3-540-36212-8

8. Portes de Albuquerque, M.; Esquef, I.A.; Gesualdi Mello, A.R.; Portes de Albuquerque, M. (2004). Image thresholding using Tsallis entropy. Pattern Recognition Letters, 25, 1059-1065. DOI: $10.1016 /$ j.patrec.2004.03.003

9. Sparavigna, A.C. (2015). On the generalized additivity of Kaniadakis entropy. International Journal of Sciences, 4(2), 44-48. http://www.ijsciences.com/pub/article/627

10. Rényi, A. (1970). Probability Theory, North-Holland: Amsterdam. ISBN 10: 0720423600, ISBN 13: 9780720423600

11. Maszczyk, T.; Duch, W. (2008). Comparison of Shannon, Rényi and Tsallis entropy used in decision trees. Lecture Notes in Computer Science, 5097, 643-651. DOI: 10.1007/978-3-54069731-2 6

12. Havrda, J.; Charvát, F. (1967). Quantification methods of classification processes: concept of structural alpha-entropy. Kybernetica (Prague), 3, 95-100.

13. Sahoo, P.K.; Arora, G. (2006). Image thresholding using two-dimensional Tsallis-HavrdaCharvát entropy. Pattern Recognition Letters, 27, 520-528. DOI: 10.1016/j.patrec.2005.09.017 
14. Sezgin, M.; Sankur, B. (2004). Survey over image thresholding techniques and quantitative performance evaluation. Journal of Electronic Imaging, 13, 146-165. DOI: 10.1117/1.1631316

15. Kapur, J.N.; Sahoo, P.K.; Wong, A.K.C. (1985). A new method for gray-level picture thresholding using the entropy of the histogram. Comput. Vision Graphics Image Process., 29, 273-285. DOI: 10.1016/0734-189X(85)90125-2

16. Shitong, Wang; Chung, F.L. (2005). Note on the equivalence relationship between Rényi-entropy based and Tsallis-entropy based image thresholding. Pattern Recognition Letters, 26, 2309-2312. DOI: $10.1016 /$ j.patrec.2005.03.027

17. Sahoo, P.; Wilkins, C.; Yeager, J. (1997). Thresholding selection using Rényi's entropy. Pattern Recognition, 30, 71-84. DOI: 10.1016/S0031-3203(96)00065-9

18. Sparavigna, A.C. (2015). Tsallis Entropy in bi-level and multi-level image thresholding. International Journal of Sciences, 4(1), 40-49. http://www.ijsciences.com/pub/article/613

19. Zhang, Y.; Wu, L. (2011). Optimal multi-level thresholding based on maximum Tsallis entropy via an artificial bee colony approach. Entropy, 13, 841-859. DOI: 10.3390/e13040841

20. Sathya, P.D.; Kayalvizhi, R. (2010). PSO-based Tsallis thresholding selection procedure for image segmentation. International Journal of Computer Applications, 5, 39-46.

21. Manikantan, K.; Arun, B.V.; Yaradoni, D.K.S. (2012). Optimal multilevel thresholds based on Tsallis entropy method using golden ratio particle swarm optimization for improved image segmentation. Procedia Engineering, 30, 364-371. DOI: 10.1016/j.proeng.2012.01.873

22. El-Sayed, M.A.; Abdel-Khalek, S.; Abdel-Aziz, E. (2011). Study of efficient technique based on 2D Tsallis entropy for image thresholding. International Journal on Computer Science and Engineering, 3, 3125-3138.

23. Kachouie, N.N.; Fieguth, P.; Ramunas, J.; Jervis, E. (2006). A statistical thresholding method for cell tracking. IEEE International Symposium on Signal Processing and Information Technology, August 2006, 222-227. DOI: 10.1109/ISSPIT.2006.270801

24. Seetharama Prasad, M.; Radha Krishna, P. (2013). A novel q-parameter automation in Tsallis entropy for image segmentation. International Journal of Computer Applications, 70, 48-53. DOI: $10.5120 / 12042-8120$

25. Elaraby, A.E.A.; El-Owny; Hassan Badry M. Ahmed; Heshmat, M.; Hassaballah, M.; Abel Rardy, A.S. (2013). A novel algorithm for edge detection of noisy medical images. International Journal of Signal Processing, Image Processing and Pattern Recognition, 6, 365-374.

26. Chaira, T. (2015). Medical Image Processing: Advanced Fuzzy Set Theoretic Techniques. CRC Press, Taylor \& Francis Group. ISBN 9781498700450

27. Weili, S.; Yu, M.; Zhanfang, C.; Hongbiao, Z. (2009). Research of automatic medical image segmentation algorithm based on Tsallis entropy and improved PCNN. International Conference on Mechatronics and Automation, ICMA, 9-12 August 2009, 1004-1008. DOI: 10.1109/ICMA.2009.5246315

28. Zhang, Y.; Wu, L. (2008). Pattern recognition via PCNN and Tsallis entropy. Sensors, 8, 75187529. DOI: $10.3390 / \mathrm{s} 8117518$

29. Ramirez-Villegas, J.F.; Ramirez-Moreno, D.F. (2012). Wavelet packet energy, Tsallis entropy and statistical parameterization for support vector-based and neural-based classification of mammographic regions. Neurocomputing, 77, 82-100. DOI: 10.1016/j.neucom.2011.08.015

30. El-Sayed, M.A. (2011). A new algorithm based entropic threshold for edge detection in images. IJCSI - International Journal of Computer Science Issues, 8, 71-78.

31. Khader, M.; Ben Hamza, A. (2012). An information- theoretic method for multimodality medical image registration. Expert Systems with Applications, 39, 5548-5556. DOI: 10.1016/j.eswa.2011.11.064

32. Zhang, Y.; Wu, L. (2012). Rigid image registration by PSOSQP algorithm. Advances in Digital Multimedia, 1, 4-8.

33. Mohanalin; Beenamol; Kalra, P.K.; Kumar, N. (2010). A novel automatic microcalcification detection technique using Tsallis entropy \& a type II fuzzy index. Computers and Mathematics with Applications, 60, 2426-2432. DOI: 10.1016/j.camwa.2010.08.038 
34. Hussain M. (2014). Mammogram enhancement using lifting dyadic wavelet transform and normalized Tsallis entropy. Journal of Computer Science and Technology, 29, 1048-1057. DOI: 10.1007/s11390-014-1489-7

35. Fabbri, R.; Gonçalves, W.N.; Lopes, F.J.P.; Bruno, O.M. (2012). Multi-q pattern analysis: A case study in image classification. Physica A: Statistical Mechanics and its Applications, 391, 44874496. DOI: 10.1016/j.physa.2012.05.001

36. Chibelushi, C.C.; Bourel, F. (2003). Facial expression recognition: A brief tutorial overview. CVonline: Compendium of Computer Vision, 9, on line.

37. Liao, S.; Fan, W.; Chung, A.C.; Yeung, D.Y. (2006). Facial expression recognition using advanced local binary patterns, Tsallis entropies and global appearance features. In Image Processing, 2006 IEEE International Conference on, 665-668. DOI: 10.1109/ICIP.2006.312418

38. Huang, D.; Shan, C.; Ardabilian, M.; Wang, Y.; Chen, L. (2011). Local binary patterns and its application to facial image analysis: a survey. Systems, Man, and Cybernetics, Part C: Applications and Reviews, IEEE Transactions on, 41, 765-781. DOI: 10.1109/TSMCC.2011.2118750

39. A. Wilson, Using fuzzy logic in image processing. Available online: http://www.visionsystems.com/index.html (accessed on 31.1.2015).

40. Zadeh, L. A. (1965). Fuzzy sets. Information and control, 8, 338-353.

41. Tizhoosh, H. R. (2005). Image thresholding using type II fuzzy sets. Pattern recognition, 38, 2363-2372. DOI: 10.1016/j.patcog.2005.02.014

42. Bustince Sola, H.; Barrenechea, E.; Pagola, M.; Fernandez, J.; Sanz, J.A. (2010). Comment on: "Image thresholding using type II fuzzy sets". Importance of this method. Pattern Recognition, 43, 3188-3192. DOI: 10.1016/j.patcog.2010.04.005

43. Tang, Y.; Di, Q.; Guan, X.; Liu, F. (2008). Threshold selection based on fuzzy Tsallis entropy and particle swarm optimization. NeuroQuantology, 6, 412-419. DOI: 10.14704/nq.2008.6.4.197 